\title{
Ground Penetrating Radar Exploration for Ancient Mausoleum at Mangshan Area
}

\author{
Wei Sun ${ }^{1,2}$, Xiaobo Huang ${ }^{1}$ \\ 1 Institute of Surveying and Mapping, \\ Information Engineering University \\ Zhengzhou, China \\ 2 Digital City Engineering Research Center Co., LTD \\ Suzhou, China \\ wsun9@163.com
}

\author{
Hui Yan, Xianqiu Wang \\ The Luoyang Municipal Institute of \\ Cultural Relics and Archaeology \\ Luoyang, China \\ sw8019@163.com
}

\begin{abstract}
Ground penetration radar (GPR) is a geophysical method that is commonly used in archaeological and forensic investigations, including the determination of the exact location of mausoleums. In this paper, a GPR case history at archaeological site is presented. The results show some very successful studies with radar to remotely sense and define subsurface archaeology. It is worthy to mention that, the excavations and location of the mausoleum matched strongly with the GPR results.
\end{abstract}

Keywords-GPR; Archaeology; Exploration; Mangshan

\section{INTRODUCTION}

Solving subsurface problems in the field of archaeology without destructively intervening with the buried materials has become a prime focus of the archaeological community. Archaeological excavations are time consuming, and, generally, not a cost-effective method for undertaking "blind" reconnaissance-type site investigations. In many cases, significant savings can be realized if exploratory excavations are sited on the basis of "leads" provided by non-invasive subsurface geophysical imaging technologies[1]-[5].

The first application of GPR in archaeology was initiated soon after the first commercial equipment became available in the 1970s. A variety of GPR case histories were published in the 1980s and 1990s. These early GPR studies were primarily concerned with the discovery of buried features within known sites rather than imaging them. The early surveys used only paper records of the real-time GPR survey and never had the ability to perform any post processing on the data. Nonetheless, great efforts were used by the early investigators to create maps of subsurface anomalies by hand-contouring locations of continuous anomalies mapped in the field. These early crude maps have since been replaced by computer-generated time slice images.

Mangshan area is the largest mausoleum in the country, brings together Zhou, Han, Wei and Western Jin, Northern Wei, Sui, Tang, Five Dynasties, Song, Jin, Yuan, Ming and Qing period, various types of ancient mausoleums. Estimated that hundreds of thousands of mausoleums, known as the "no cattle lies". Mangshan is a famous treasure trove of underground cultural relics. Tens of thousands have high historical, artistic or scientific value of precious cultural relics have been unearthed in the past Mangshan. Many well-known domestic and foreign museums, galleries and collections have the Mangshan unearthed cultural relics.

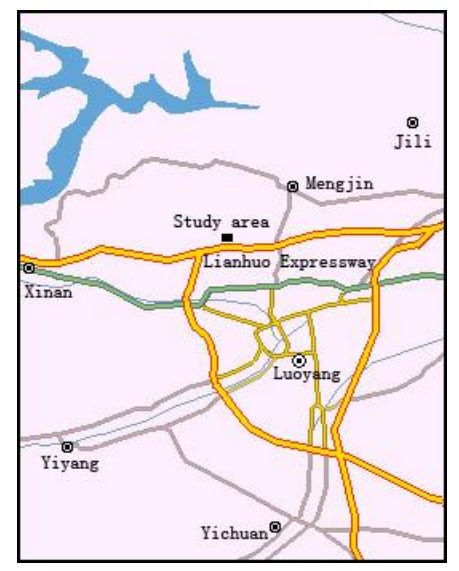

Figure 1. Location map of the study area.

In this paper we will briefly introduce the GPR field methods for archaeological investigation and look at a case GPR mausoleum that involves GPR imaging techniques.

\section{PRINCIPLE OF GPR MEASUREMENTS}

The GPR is a broad-spectrum $(1 \mathrm{MHz}-1 \mathrm{GHz})$ electromagnetic technology used in detecting reflections from subsurface features [6]. The range of applications for GPR methods is wide and the sophistication of signal recovery techniques, hardware designs and operating practices is increasing as the technology matures. As a relatively new geophysical method, it plays an important role in the superficial stratum detection, due to its easy operation, high automation, high resolution images and reliable interpretation results. It has successfully used in various fields as landmine detection, municipal pipelines mapping, non-destructive roads testing, archaeological detectors and etc. The GPR uses high frequency electromagnetic waves to realize the subsurface. For the dielectric constant differences of different media, the

Sponsored by Natural Science Fund of Jiangsu Province (BK2011343),

Government Assistance Plan of Suzhou Industrial Park Science and

Technology Agency (SG201030). 
underground media can be detected using their reflection image. Currently the dual-antenna GPR usually takes the profiling measurement method.

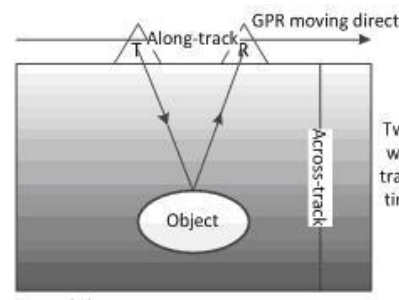

(a) Reflection measurement mode

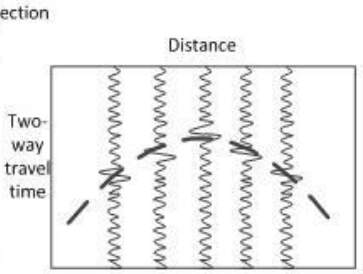

(b) Imaging schematic
Figure 2. Radar scan generation.

The profiling method is a synchronous measuring approach by moving the transmitting antenna $(\mathrm{T})$ and receiving antenna (R) at constant interval along the survey line. One record is gotten when the transmitter and the receiver synchronously move once. When the transmitter and the receiver simultaneously move along the survey lines, a time profile GPR image can be composed by sequential records. The abscissa of the image is the antenna position along the surface measuring line. The vertical axis is the two-way travel time of the signals traveling from the transmitter to the receiver reflector through the underground interface. Such records can accurately reflect the undulations of each reflector directly below the survey line, as shown in Fig. 2.

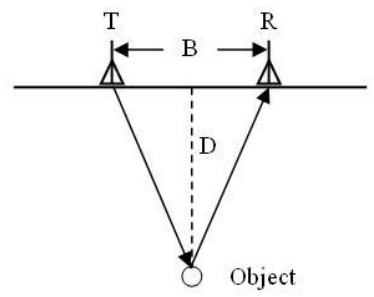

Figure 3. The buried target of GPR measurement

It can be seen form Fig. 3 that the two-way travel time of the radar pulse is calculated as

$$
t=\frac{1}{v} \sqrt{4 D^{2}+B^{2}}
$$

When the transmitter and the receiver is combined $\mathrm{B}=0$, then (2) can be simplified as

$$
D=\frac{t v}{2}
$$

\section{FIELD METHODS FOR ARCHAEOLOGICAL ACQUISITION}

For most archaeological surveys with GPR, detection is the most important aspect of the investigation. When a sufficient density of profiles is recorded across a site, structural information can also be obtained regarding the buried features.

\section{A. Survey description}

The survey region was $15 \mathrm{~m} \times 30 \mathrm{~m}$ with a grid pattern survey. Trace spacing was $2 \mathrm{~m}$, and the total time window was
$300 \mathrm{~ns}$, with $0.36 \mathrm{~ns}$ of time sampling interval per trace on each profile. The radar data were acquired using the RIS GPR instrument. The $40 \mathrm{MHz}$ antenna was used in the field survey with the survey point mode data collection. The survey line layout is shown in Fig. 4.

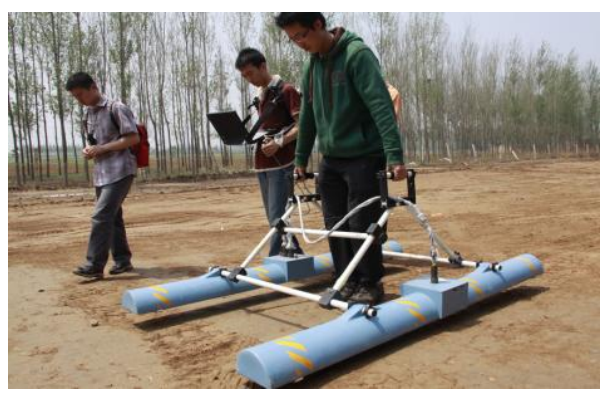

Figure 4. Radar survey at Mangshan area.

The main consideration in laying out survey lines is the line spacing. Generally speaking, the smaller the survey line spacing is, the more accurate the measurement is. But at the same time the efficiency should be taken into account.

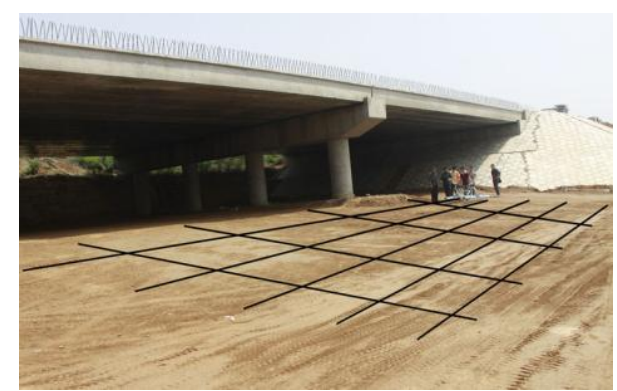

Figure 5. The GPR survey line layout diagram.

In archaeological investigation we prefer to choose a line density and profile direction that may yield the most successful imaging. In surveying mausoleum sites, for example, where there is some initial knowledge that the mausoleums are running in a roughly north-south direction, we will normally orient the line taking in an east-west direction. This will yield the best possible chance of traversing the mausoleum. If a profile spacing of say $5 \mathrm{~m}$ were applied, there is a possibility of missing a mausoleum if one were to profile parallel to the long axis, because the mausoleum may be less than $5 \mathrm{~m}$ wide. Taking data perpendicular to the longer axis of the mausoleum, or for that matter any archaeological structure, will aid detection. Recording of profiles in orthogonal directions will insure that a buried artifact will have been traversed and increase the probability of detection if the line density is at least half the smallest horizontal dimension of the buried targets.

\section{B. Depth Determination}

Determining the depth of a GPR survey can be accomplished by several methods:

1. making laboratory measurements of the dielectric and conductivity; 
2. placement of a known object at depth to measure the two-way travel time;

3. wide angle measurement using separated transmitterreceiver antenna;

4. matching the shape of hyperbolas detected on the GPR radargram.

In this paper we use to extract the B-scan feature to estimate the depth from the hyperbolic method.

The method involves matching of hyperbola observed on recorded radar image and is the best method for remotely measuring the electrical properties of material. The width of hyperbolas recorded off small (point source) objects is the "DNA" of the ground which provides an estimate of the microwave velocity. The narrower the hyperbola, the slower the microwave velocity is; the wider the hyperbola, the faster the microwave velocity of the ground surrounding the target is.

\section{SURVEY DATA PROCESSING}

In general, most published GPR data have been processed and presented in either A-scan, B-scan or C-scan form [7]. The processes applied to each data format can be broadly classified as follows:

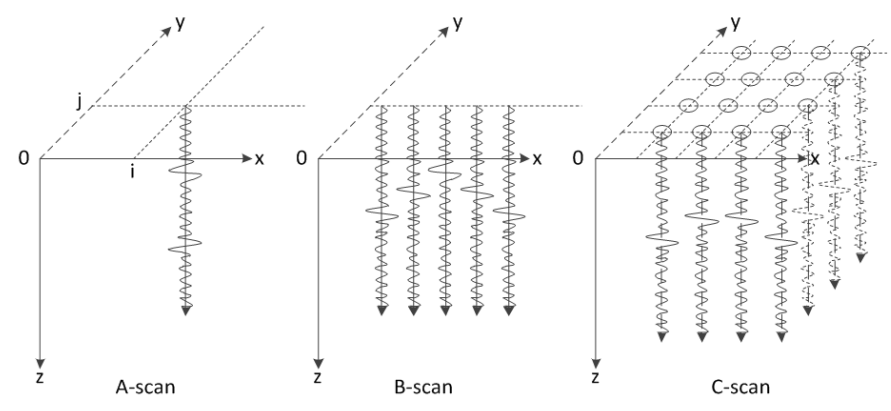

Figure 6. Coordinate system for scan description.

(a) A-scan processing.

(b) B-scan processing. Note that the dimensions can be considered interchangeable, that is, the same processes may be useful for $\mathrm{x}-\mathrm{z}, \mathrm{x}-\mathrm{y}$ or $\mathrm{y}-\mathrm{z}$ planes.

(c) C-scan processing. Note that the spatial threedimensional data can be used to reconstruct representations of three-dimensional images.

The raw data collected by the GPR field work should go through data processing to get measurable images. The raw data contains both useful information and various noises. In some cases useful information may be hidden from the noises. The purpose of the data processing is to suppress noise, enhance signal and improve Signal Noise Ratio (SNR), in order to extract velocity, amplitude, frequency, phase and other information from the data [8]. The flowchart of GPR data processing is shown in Fig. 7.

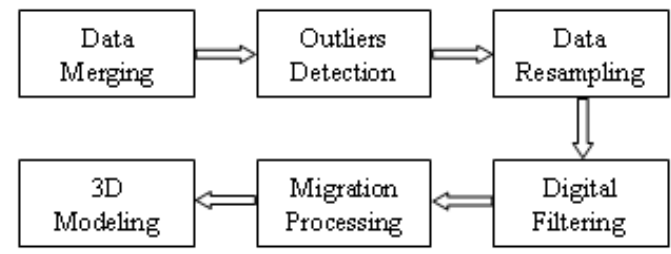

Figure 7. The flowchart of GPR data processing.

In order to remove the disturbing signals in GPR measured data, the appropriate digital filtering method should be taken in the data processing. According to test results, the spectrum of noise components lies both in the low frequency and the high frequency components. Therefore, the bandpass filter should be used to filter out noises in data processing [9]. Nonlinear filtering method is also used to highlight the pipeline's radar reflection.

We acquired and processed parallel 2D GPR profile data. They are shown as grey scale B-scans to illustrate the effect of applying various algorithms on a radar image of two pipelines. In Figure 8 we show a line of data. The left vertical axis is the depth information calculated by the velocity. Abscissa records the position of the radar antenna along the survey line.

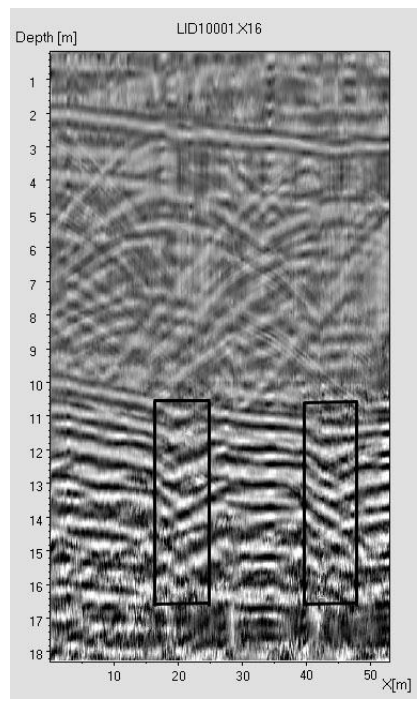

Figure 8. Radar sections with walls marked.

From radar image it can be clearly identified the clear anomaly event is present related to the wall. Within the scope of the survey line, the depth of the two walls is averaged 10.5 $\mathrm{m}$. About 10 meters from the image it can be seen the thickness of the cover soil. The cover soil is two parts. The ground to a depth of 2 meters mound should be naturally formed. Part of the cover soil of the depth of $2 \mathrm{~m}$ to $10 \mathrm{~m}$ is the original mound.

In general, the smallest detectable size of archaeological materials is in general dependent on the frequency of the transmitting antenna. Typical frequencies for archaeological investigation range from about 200 to $800 \mathrm{MHz}$; however, even a low-frequency antenna on the order of $20 \mathrm{MHz}$ might be used to discover structures buried below $15 \mathrm{~m}$ and $4 \mathrm{GHz}$ antenna might be used to measure shallow features a few centimeters thick. 
The photograph of excavated mausoleum is shown in Fig. 9. It is worthy to mention that, the excavations and location of the mausoleum matched strongly with the GPR results.

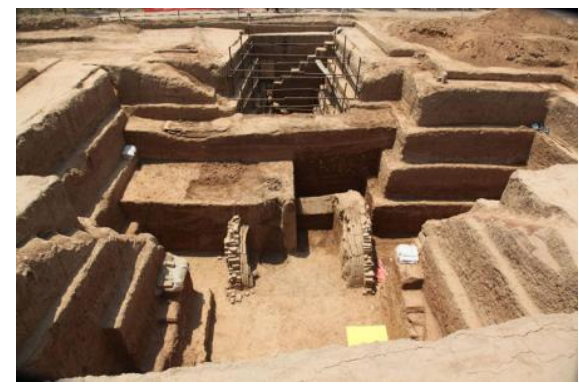

Figure 9. Photograph of excavated mausoleum.

GPR imaging has been able to help archaeologists determine the major structural layout of the mausoleum site. All was accomplished without any extensive excavation other than to verify structures and target potential sites with the most potential for recovering artifacts. The clarity in the GPR images on use in Mangshan sites from the mausoleum site show the potential of this remote sensing tool.

\section{CONCLUSION}

The science to study, measure and quantify archaeological structures remotely has been designated as the field of archaeology. Remotely detecting archaeological structures is very important because excavation of a site can inadvertently destroy essential archaeological evidence which can then never be recovered. Because of the successful application of a variety of geophysical tools, and in particular GPR to probe beneath the ground, many archaeologists now regularly initiate geophysical surveys before studying or excavating potential sites. The application of GPR in archaeology has ranged from studying protected sites which can never be excavated, to using GPR to quickly and cost-effectively plan and carry out mitigation projects. GPR surveys at sites that are impacted by development fall under the category of rescue archaeology.
This is the largest growing segment of the GPR applications in archaeology, and these kinds of surveys are expedited by a growing number of geotechnical consulting firms.

\section{ACKNOWLEDGMENT}

We would like to thank the Beijing PT Equipment Co., Ltd for their help in providing ground penetration radar system and express our deep gratitude to Hubin Bai for his survey.

\section{REFERENCES}

[1] Harry M. Jol, Ground Penetrating radar theory and applications, Elsevier Science, Radarweg 29, PO Box 211, $1000 \mathrm{AE}$ Amsterdam, The Netherlands, The Boulevard, Langford Lane, Kidlington, Oxford OX5 1GB, UK, 2009.

[2] Clark, J.A., "Seeing Beneath the Soil: Prospection Methods in Archaeology," B. T. Batsford, London, 1990.

[3] Scollar, I., Tabbagh, A., Hesse, A., Herzog, I., "Archaeological Prospecting and Remote Sensing," Cambridge University Press, Cambridge0 52132050 X. 674 pages, 1990.

[4] Gaffney, C., Gater, J., "Revealing the Buried Past: Geophysics for Archaeologists," Tempus Publishing Ltd, Gloucestershire07524-2556-0. 192 pages, 104 figures, 24 plates, 2003.

[5] Fathy A. Shaaban, Abbas M. Abbas, Magdy A. Atya, Mahfouz A. Hafez, "Ground-penetrating radar exploration for ancient monuments at the Valley of Mummies -Kilo 6, Bahariya Oasis, Egypt," Journal of Applied Geophysics, 2009, no. 68, pp. 194202.

[6] Li Daxin, Application of Ground Penetrating Radar, Beijing:China Geologic Press, 1994, pp. 1-2.

[7] D. J. Daniels, Ground Penetrating Radar, 2nd ed. ser. IEE Radar, Sonar, Navigation And Avionics Series 15, N. Stewart and H. Griffiths, Eds. London, U.K.: IEE press, 2004.

[8] Sun Wei, Xu Qing, Shang Xiangyang etc, “Application of Ground Penetrating Radar with GPS in Underwater Topographic Survey," AIMSEC 2011, vol. 3, Zhengzhou, 2011, pp.19461949.

[9] Sun Wei, Xu Qing, Zhang Heng etc, "Research on Detection and Visualization of Underground Pipelines," RSETE 2012, vol. 4, Nanjing, 2012, pp.2216-2219. 